\title{
Screening for Breast Cancer: U.S. Preventive Services Task Force Recommendation Statement
}

\author{
Albert L. Siu, MD, MSPH, on behalf of the U.S. Preventive Services Task Force*
}

Description: Update of the 2009 U.S. Preventive Services Task Force (USPSTF) recommendation on screening for breast cancer.

Methods: The USPSTF reviewed the evidence on the following: effectiveness of breast cancer screening in reducing breast cancer-specific and all-cause mortality, as well as the incidence of advanced breast cancer and treatment-related morbidity; harms of breast cancer screening; test performance characteristics of digital breast tomosynthesis as a primary screening strategy; and adjunctive screening in women with increased breast density. In addition, the USPSTF reviewed comparative decision models on optimal starting and stopping ages and intervals for screening mammography; how breast density, breast cancer risk, and comorbidity level affect the balance of benefit and harms of screening mammography; and the number of radiation-induced breast cancer cases and deaths associated with different screening mammography strategies over the course of a woman's lifetime.

Population: This recommendation applies to asymptomatic women aged 40 years or older who do not have preexisting breast cancer or a previously diagnosed high-risk breast lesion and who are not at high risk for breast cancer because of a known underlying genetic mutation (such as a BRCA1 or BRCA2 gene mutation or other familial breast cancer syndrome) or a history of chest radiation at a young age.
Recommendations: The USPSTF recommends biennial screening mammography for women aged 50 to 74 years. (B recommendation)

The decision to start screening mammography in women prior to age 50 years should be an individual one. Women who place a higher value on the potential benefit than the potential harms may choose to begin biennial screening between the ages of 40 and 49 years. (C recommendation)

The USPSTF concludes that the current evidence is insufficient to assess the balance of benefits and harms of screening mammography in women aged 75 years or older. (I statement)

The USPSTF concludes that the current evidence is insufficient to assess the benefits and harms of digital breast tomosynthesis (DBT) as a primary screening method for breast cancer. (I statement)

The USPSTF concludes that the current evidence is insufficient to assess the balance of benefits and harms of adjunctive screening for breast cancer using breast ultrasonography, magnetic resonance imaging (MRI), DBT, or other methods in women identified to have dense breasts on an otherwise negative screening mammogram. (I statement)

Ann Intern Med. 2016;164:279-296. doi:10.7326/M15-2886 www.annals.org For author affiliation, see end of text.

This article was published at www.annals.org on 12 January 2016

* For members of the USPSTF, see the Appendix (available at www.annals.org).
$\mathbf{T}^{\text {he }}$ he U.S. Preventive Services Task Force (USPSTF) makes recommendations about the effectiveness of specific preventive care services for patients without related signs or symptoms.

It bases its recommendations on the evidence of both the benefits and harms of the service and an assessment of the balance. The USPSTF does not consider the costs of providing a service in this assessment.

The USPSTF recognizes that clinical decisions involve more considerations than evidence alone. Clinicians should understand the evidence but individualize decision making to the specific patient or situation. Similarly, the USPSTF notes that policy and coverage decisions involve considerations in addition to the evidence of clinical benefits and harms.

\section{SumMaRy OF RECOMMENDATIONS AND EVIDENCE}

The USPSTF recommends biennial screening mammography for women aged 50 to 74 years. (B recommendation)
The decision to start screening mammography in women prior to age 50 years should be an individual one. Women who place a higher value on the potential benefit than the potential harms may choose to begin biennial screening between the ages of 40 and 49 years. (C recommendation)

- For women who are at average risk for breast cancer, most of the benefit of mammography results from biennial screening during ages 50 to 74 years. Of all of the age groups, women aged 60 to 69 years are

\section{See also:}

Related articles . . 205, 215, 226, 236, 244, 256, 268

Editorial comments .............. 301, 303

Summary for Patients. . . . . . . . . . . . . . I-28

Web-Only

Consumer Fact Sheet 
most likely to avoid breast cancer death through mammography screening. While screening mammography in women aged 40 to 49 years may reduce the risk for breast cancer death, the number of deaths averted is smaller than that in older women and the number of false-positive results and unnecessary biopsies is larger. The balance of benefits and harms is likely to improve as women move from their early to late 40 s.

- In addition to false-positive results and unnecessary biopsies, all women undergoing regular screening mammography are at risk for the diagnosis and treatment of noninvasive and invasive breast cancer that would otherwise not have become a threat to their health, or even apparent, during their lifetime (known as "overdiagnosis"). Beginning mammography screening at a younger age and screening more frequently may increase the risk for overdiagnosis and subsequent overtreatment.

- Women with a parent, sibling, or child with breast cancer are at higher risk for breast cancer and thus may benefit more than average-risk women from beginning screening in their 40s.

Go to the Clinical Considerations section for information on implementation of the $\mathrm{C}$ recommendation.

The USPSTF concludes that the current evidence is insufficient to assess the balance of benefits and harms of screening mammography in women aged 75 years or older. (I statement)

The USPSTF concludes that the current evidence is insufficient to assess the benefits and harms of digital breast tomosynthesis (DBT) as a primary screening method for breast cancer. (I statement)

The USPSTF concludes that the current evidence is insufficient to assess the balance of benefits and harms of adjunctive screening for breast cancer using breast ultrasonography, magnetic resonance imaging (MRI), DBT, or other methods in women identified to have dense breasts on an otherwise negative screening mammogram. (I statement)

These recommendations apply to asymptomatic women aged 40 years or older who do not have preexisting breast cancer or a previously diagnosed highrisk breast lesion and who are not at high risk for breast cancer because of a known underlying genetic mutation (such as a BRCA1 or BRCA2 gene mutation or other familial breast cancer syndrome) or a history of chest radiation at a young age.

See Figure 1 for a summary of the recommendation and suggestions for clinical practice.

Appendix Table 1 describes the USPSTF grades, and Appendix Table 2 describes the USPSTF classification of levels of certainty about net benefit (both tables are available at www.annals.org).

\section{RATiOnALE \\ Importance}

Breast cancer is the second-leading cause of cancer death among women in the United States. In 2015, an estimated 232000 women were diagnosed with the disease and 40000 women died of it. It is most frequently diagnosed among women aged 55 to 64 years, and the median age of death from breast cancer is 68 years (1).

\section{Benefit and Harms of Screening and Early Treatment}

The USPSTF found adequate evidence that mammography screening reduces breast cancer mortality in women aged 40 to 74 years. The number of breast cancer deaths averted increases with age; women aged 40 to 49 years benefit the least and women aged 60 to 69 years benefit the most. Age is the most important risk factor for breast cancer, and the increased benefit observed with age is at least partly due to the increase in risk. Women aged 40 to 49 years who have a firstdegree relative with breast cancer have a risk for breast cancer similar to that of women aged 50 to 59 years without a family history. Direct evidence about the benefits of screening mammography in women aged 75 years or older is lacking.

The USPSTF found adequate evidence that screening for breast cancer with mammography results in harms for women aged 40 to 74 years. The most important harm is the diagnosis and treatment of noninvasive and invasive breast cancer that would otherwise not have become a threat to a woman's health, or even apparent, during her lifetime (that is, overdiagnosis and overtreatment). False-positive results are common and lead to unnecessary and sometimes invasive follow-up testing, with the potential for psychological harms (such as anxiety). False-negative results (that is, missed cancer) also occur and may provide false reassurance. Radiation-induced breast cancer and resulting death can also occur, although the number of both of these events is predicted to be low.

The USPSTF found inadequate evidence on the benefits and harms of DBT as a primary screening method for breast cancer. Similarly, the USPSTF found inadequate evidence on the benefits and harms of adjunctive screening for breast cancer using breast ultrasonography, MRI, DBT, or other methods in women identified to have dense breasts on an otherwise negative screening mammogram. In both cases, while there is some information about the accuracy of these methods, there is no information on the effects of their use on health outcomes, such as breast cancer incidence, mortality, or overdiagnosis rates.

\section{USPSTF Assessment}

The USPSTF concludes with moderate certainty that the net benefit of screening mammography in women aged 50 to 74 years is moderate.

The USPSTF concludes with moderate certainty that the net benefit of screening mammography in the general population of women aged 40 to 49 years, while positive, is small.

The USPSTF concludes that the evidence on mammography screening in women age 75 years and older is insufficient, and the balance of benefits and harms cannot be determined. 


\section{Annals of Internal Medicine}

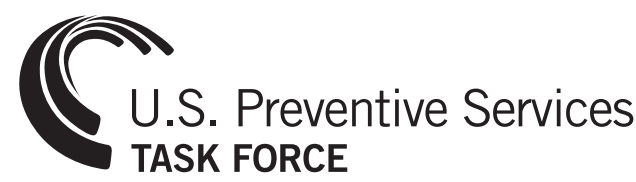

Primary Screening for Breast Cancer With Conventional Mammography

\begin{tabular}{|c|c|c|c|}
\hline Population & Women aged 40 to $49 \mathrm{y}$ & Women aged 50 to $74 \mathrm{y}$ & Women aged $\geq 75$ y \\
\hline Recommendation & $\begin{array}{c}\text { The decision to start screening should be } \\
\text { an individual one. } \\
\text { Grade: } \mathrm{C}\end{array}$ & $\begin{array}{c}\text { Screen every } 2 \text { years. } \\
\text { Grade: B }\end{array}$ & $\begin{array}{l}\text { No recommendation. } \\
\text { Grade: I statement } \\
\text { (insufficient evidence) }\end{array}$ \\
\hline
\end{tabular}

\begin{tabular}{|c|c|c|c|}
\hline Risk Assessment & \multicolumn{3}{|c|}{$\begin{array}{c}\text { These recommendations apply to asymptomatic women aged } \geq 40 \text { y who do not have preexisting breast cancer or a previously } \\
\text { diagnosed high-risk breast lesion and who are not at high risk for breast cancer because of a known underlying genetic mutation } \\
\text { (such as a BRCA1 or BRCA2 gene mutation or other familial breast cancer syndrome) or a history of chest radiation at a young age. } \\
\text { Increasing age is the most important risk factor for most women. }\end{array}$} \\
\hline Screening Tests & \multicolumn{3}{|c|}{$\begin{array}{l}\text { Conventional digital mammography has essentially replaced film mammography as the primary method for breast cancer screening } \\
\text { in the United States. Conventional digital screening mammography has about the same diagnostic accuracy as film overall, although } \\
\text { digital screening seems to have comparatively higher sensitivity but the same or lower specificity in women age }<50 \mathrm{y} \text {. }\end{array}$} \\
\hline $\begin{array}{l}\text { Starting and } \\
\text { Stopping Ages }\end{array}$ & \multicolumn{3}{|c|}{$\begin{array}{l}\text { For women who are at average risk for breast cancer, most of the benefit of mammography results from biennial screening during } \\
\text { ages } 50 \text { to } 74 \mathrm{y} \text {. While screening mammography in women aged } 40 \text { to } 49 \mathrm{y} \text { may reduce the risk for breast cancer death, the } \\
\text { number of deaths averted is smaller than that in older women and the number of false-positive results and unnecessary biopsies is } \\
\text { larger. The balance of benefits and harms is likely to improve as women move from their early to late } 40 \text { s. }\end{array}$} \\
\hline Screening Interval & \multicolumn{3}{|c|}{ For most women, biennial mammography screening provides the best overall balance of benefit and harms. } \\
\hline $\begin{array}{l}\text { Balance of Benefits } \\
\text { and Harms }\end{array}$ & $\begin{array}{l}\text { The net benefit of screening mammography } \\
\text { in women aged } 40 \text { to } 49 \mathrm{y} \text {, while positive, is } \\
\text { small. }\end{array}$ & $\begin{array}{l}\text { The net benefit of screening } \\
\text { mammography in women aged } 50 \\
\text { to } 74 \text { y is moderate. }\end{array}$ & $\begin{array}{l}\text { Evidence on mammography screening in } \\
\text { women aged } \geq 75 y \text { is insufficient, and the } \\
\text { balance of benefits and harms cannot be } \\
\text { determined. }\end{array}$ \\
\hline $\begin{array}{l}\text { Other Relevant } \\
\text { USPSTF } \\
\text { Recommendations }\end{array}$ & \multicolumn{3}{|c|}{$\begin{array}{l}\text { The USPSTF has made recommendations about the use of medications to reduce women's risk for breast cancer, as well as risk } \\
\text { assessment, genetic counseling, and genetic testing for BRCA1- or BRCA2-related cancer (including breast cancer). These } \\
\text { recommendations are available on the USPSTF Web site (www.uspreventiveservicestaskforce.org). }\end{array}$} \\
\hline
\end{tabular}

Screening for Breast Cancer With Methods Other Than Conventional Mammography

\begin{tabular}{|l|c|c|}
\hline Screening Method & Primary screening with DBT & $\begin{array}{c}\text { Adjunctive screening with breast ultrasonography, MRI, DBT, } \\
\text { or other methods in women who have dense breasts }\end{array}$ \\
\hline Recommendation & $\begin{array}{c}\text { No recommendation. } \\
\text { Grade: I statement } \\
\text { (insufficient evidence) }\end{array}$ & $\begin{array}{c}\text { No recommendation. } \\
\text { Grade: I statement } \\
\text { (insufficient evidence) }\end{array}$ \\
\hline
\end{tabular}

\begin{tabular}{|c|c|c|}
\hline Benefits & $\begin{array}{l}\text { From the limited data available, DBT seems to reduce recall } \\
\text { rates (i.e., follow-up for additional imaging or testing) and } \\
\text { increase cancer detection rates compared with conventional } \\
\text { digital mammography alone. }\end{array}$ & $\begin{array}{l}\text { Limited data suggests that ultrasonography or MRI will detect } \\
\text { additional breast cancer in women who have dense breasts. } \\
\text { DBT also detects additional breast cancer in the short term. }\end{array}$ \\
\hline Harms & $\begin{array}{c}\text { As currently practiced in most settings, DBT exposes women to } \\
\text { about twice the amount of radiation as conventional digital } \\
\text { mammography. Current study designs cannot determine the } \\
\text { degree to which the additional cases of cancer detected would } \\
\text { have become clinically significant (i.e., the degree of } \\
\text { overdiagnosis). }\end{array}$ & $\begin{array}{l}\text { Most positive adjunctive breast cancer screening test results } \\
\text { are false positive. }\end{array}$ \\
\hline $\begin{array}{l}\text { Balance of Benefits } \\
\text { and Harms }\end{array}$ & $\begin{array}{l}\text { Evidence is insufficient, and the balance of benefits and harms } \\
\text { cannot be determined. }\end{array}$ & $\begin{array}{l}\text { Evidence is insufficient, and the balance of benefits and harms } \\
\text { cannot be determined. }\end{array}$ \\
\hline
\end{tabular}

For a summary of the evidence systematically reviewed in making this recommendation, the full recommendation statement, and supporting documents, please go to www.uspreventiveservicestaskforce.org.

The USPSTF concludes that the evidence on DBT as a primary screening method for breast cancer is insufficient, and the balance of benefits and harms cannot be determined.
The USPSTF concludes that the evidence on adjunctive screening for breast cancer using breast ultrasound, MRI, DBT, or other methods in women identified to have dense breasts on an otherwise negative 
Table 1. Breast Cancer Deaths Avoided (95\% Cl) per 10000 Women Screened by Repeat Screening Mammography Over 10 Years: Data From Randomized, Controlled Trials*

\begin{tabular}{llll}
\hline Variable & Ages 40-49 $\mathbf{y}$ & Ages 50-59 y & Ages 60-69 y \\
\hline Breast cancer deaths avoided & $3(0-9)$ & $8(2-17)$ & $21(11-32)$ \\
\hline
\end{tabular}

* All women did not have $100 \%$ adherence to all rounds of screening offered in the randomized, controlled trials.

screening mammogram is insufficient, and the balance of benefits and harms cannot be determined.

\section{Clinical Considerations Benefit of Screening}

The results of the meta-analysis of clinical trials from the systematic evidence review commissioned by the USPSTF are summarized in Table 1. Over a 10-year period, screening 10000 women aged 60 to 69 years will result in 21 (95\% Cl, 11 to 32) fewer breast cancer deaths. The benefit is smaller in younger women: Screening 10000 women aged 50 to 59 years will result in $8(\mathrm{Cl}, 2$ to 17$)$ fewer breast cancer deaths, and screening 10000 women aged 40 to 49 years will result in $3(\mathrm{Cl}, 0$ to 9$)$ fewer breast cancer deaths $(2,3)$. Most of these trials began enrollment more than 30 years ago, and these estimates may not reflect the current likelihood of avoiding a breast cancer death with contemporary screening mammography technology. Mammography imaging has since improved, which may result in more tumors being detected at a curable stage today than at the time of these trials. However, breast cancer treatments have also improved, and as treatment improves, the advantage of earlier detection decreases, so that some of the women who died of breast cancer in the nonscreened groups in these trials would survive today.

\section{Harms of Screening}

The most important harm of screening is the detection and treatment of invasive and noninvasive cancer that would never have been detected, or threaten health, in the absence of screening (overdiagnosis and overtreatment). Existing science does not allow for the ability to determine precisely what proportion of cancer diagnosed by mammography today reflects overdiagnosis, and estimates vary widely depending on the data source and method of calculation used $(2,4)$. In the United States, the rate of diagnosis of invasive plus noninvasive breast cancer increased by $50 \%$ during the era of mammography screening (Figure 2) (5). It is not possible to know with certainty what proportion of that increase is due to overdiagnosis and what proportion reflects other reasons for a rising incidence. If overdiagnosis is the only explanation for the increase, 1 in 3 women diagnosed with breast cancer today is being treated for cancer that would never have been discovered or caused her health problems in the absence of screening. The best estimates from randomized, controlled trials (RCTs) evaluating the effect of mammography screening on breast cancer mortality suggest that 1 in 5 women diagnosed with breast cancer over approximately 10 years will be overdiagnosed (6). Modeling studies conducted in support of this recommendation by the Cancer Intervention and Surveillance Modeling Network (CISNET) provide a range of estimates that reflect different underlying assumptions; the median estimate is that 1 in 8 women diagnosed with breast cancer with biennial screening from ages 50 to 75 years will be overdiagnosed. The rate increases with an earlier start age or with annual mammography $(7,8)$. Even with the conservative estimate of 1 in 8 breast cancer cases being overdiagnosed, for every woman who avoids a death from breast cancer through screening, 2 to 3 women will be treated unnecessarily.

The other principal harms of screening are falsepositive results, which require further imaging and often breast biopsy, and false-negative results. Table 2 summarizes the rates of these harms per screening round using registry data for digital mammography from the Breast Cancer Surveillance Consortium (BCSC), a collaborative network of 5 mammography registries and 2 affiliated sites with linkages to tumor registries across the United States $(2,9)$. (Note that Table 2 describes a different time horizon than Table 1 [per screening round rather than per decade].)

\section{When to Start Screening}

Clinical trials, observational studies, and modeling studies all demonstrate that the likelihood of avoiding a breast cancer death with regular screening mammography increases with age, and this increase in benefit likely occurs gradually rather than abruptly at any particular age. In contrast, the harms of screening mammography either remain constant or decrease with age. For example, about the same number of breast biopsies are performed as a result of screening mammography in women aged 40 to 49 years as in those aged 60 to 69 years, but many more of these biopsies will result in a diagnosis of invasive cancer in the older age group. Thus, the balance of benefit and harms improves with age (Table 3 ).

The USPSTF concludes that while there are harms of mammography, the benefit of screening mammography outweighs the harms by at least a moderate amount from age 50 to 74 years and is greatest for women in their 60s. For women in their 40s, the number who benefit from starting regular screening mammography is smaller and the number experiencing harm is larger compared with older women. For women in their 40s, the benefit still outweighs the harms, but to a smaller degree; this balance may therefore be more subject to individual values and preferences than it is in older women. Women in their 40s must weigh a very important but infrequent benefit (reduction in breast cancer deaths) against a group of 
meaningful and more common harms (overdiagnosis and overtreatment, unnecessary and sometimes invasive follow-up testing and psychological harms associated with false-positive test results, and false reassurance from false-negative test results). Women who value the possible benefit of screening mammography more than they value avoiding its harms can make an informed decision to begin screening.

Neither clinical trials nor models can precisely predict the potential benefits and harms that an individual woman can expect from beginning screening at age 40 rather than 50 years, as these data represent population effects. However, model results may be the easiest way for women to visualize the relative tradeoffs of beginning screening at age 40 versus 50 years. CISNET conducted modeling studies to predict the lifetime benefits and harms of screening with contemporary digital mammography at different starting and stopping ages and screening intervals. The models varied their assumptions about the natural history of invasive and noninvasive breast cancer and the effect of detection by digital mammography on survival. The models assumed the ideal circumstances of perfect adherence to screening and current best practices for therapy across the life span. Table 3 compares the median and range across the models for predicted lifetime benefits and harms of screening biennially from ages 50 to 74 years with screening biennially from ages 40 to 74 years. (Note that Table 3 differs from Tables 1 and 2 in terms of population metrics [per 1000 vs. 10000 women] and time horizon considered [lifetime vs. 10year or single event].)

It is, however, a false dichotomy to assume that the only options are to begin screening at age 40 or to wait until age 50 years. As women advance through their $40 \mathrm{~s}$, the incidence of breast cancer rises. The balance of benefit and harms may also shift accordingly over this decade, such that women in the latter half of the decade likely have a more favorable balance than women in the first half. Indeed, the CISNET models suggest that most of the benefit of screening women aged 40 to 49 years would be realized by starting screening at age $45(7,8)$.

Figure 2. Trends in invasive and noninvasive breast cancer incidence and mortality since the widespread introduction of screening mammography in the United States.

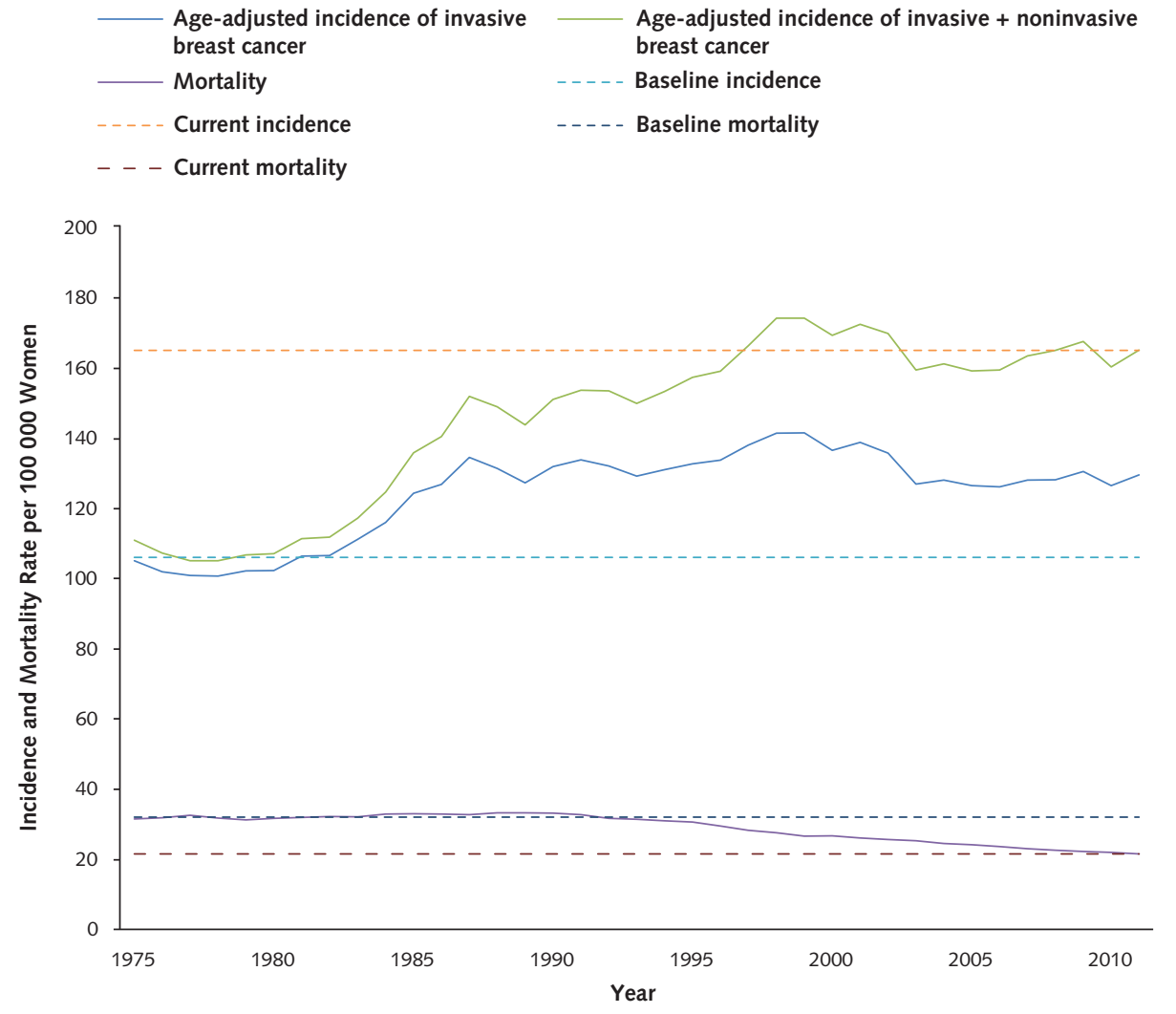

The figure depicts changes in age-adjusted invasive and combined invasive and noninvasive breast cancer incidence and mortality rates in the United States from 1975 through 2011. The baseline breast cancer incidence rate was 105 to 111 cases per 100000 women (depending on whether one considers invasive disease or invasive plus noninvasive disease together). With the widespread diffusion of mammography screening, this rate increased to 165 cases of noninvasive plus invasive disease per 100000 women in 2011 (an excess of 54 to 60 cases per 100000 women, or about a 50\% increase). Breast cancer mortality rates have declined at a slower rate, from 31 to 22 cases (or a reduction of 9 deaths) per 100000 women over the same time period. Based on data from the National Cancer Institute's Surveillance, Epidemiology, and End Results (SEER) Program (5) 
Table 2. Harms of One-Time Mammography Screening per 10000 Women Screened: Breast Cancer Surveillance Consortium Registry Data

\begin{tabular}{lllll}
\hline Variable & Ages 40-49 y & Ages 50-59 y & Ages 60-69 y & Ages 70-74 y \\
\hline False-positive test results (false alarms), $n$ & 1212 & 932 & 808 & 696 \\
Breast biopsies, $n$ & 164 & 159 & 165 & 175 \\
False-negative test results (missed cancers), $n$ & 10 & 11 & 12 & 13 \\
\hline
\end{tabular}

\section{Risk Factors That May Influence When to Start Screening}

Advancing age is the most important risk factor for breast cancer in most women, but epidemiologic data from the BCSC suggest that having a first-degree relative with breast cancer is associated with an approximately 2 -fold increased risk for breast cancer in women aged 40 to 49 years $(2,9)$. Further, the CISNET models suggest that for women with about a 2-fold increased risk for breast cancer, starting annual digital screening at age 40 years results in a similar harm-to-benefit ratio (based on number of false-positive results or overdiagnosed cases per 1000 breast cancer deaths avoided) as beginning biennial digital screening at age 50 years in average-risk women $(7,8)$. This approach has not been formally tested in a clinical trial; therefore, there is no direct evidence that it would result in net benefit similar to that of women aged 50 to 74 years. However, given the increased burden of disease and potential likelihood of benefit, women aged 40 to 49 years who have a known first-degree relative (parent, child, or sibling) with breast cancer may consider initiating screening earlier than age 50 years. Many other risk factors have been associated with breast cancer in epidemiologic studies, but most of these relationships are weak or inconsistent and would not likely influence how women value the tradeoffs of the potential benefits and harms of screening. Risk calculators, such as the National Cancer Institute's Breast Cancer Risk Assessment Tool (available at www.cancer.gov/BCRISKTOOL), have good calibration between predicted and actual outcomes in groups of women but are not accurate at predicting an individual woman's risk for breast cancer (10).

\section{How Often to Screen}

Once a woman has decided to begin screening, the next decision is how often to undergo screening. No clinical trials compared annual mammography with a longer interval in women of any age. In the random-

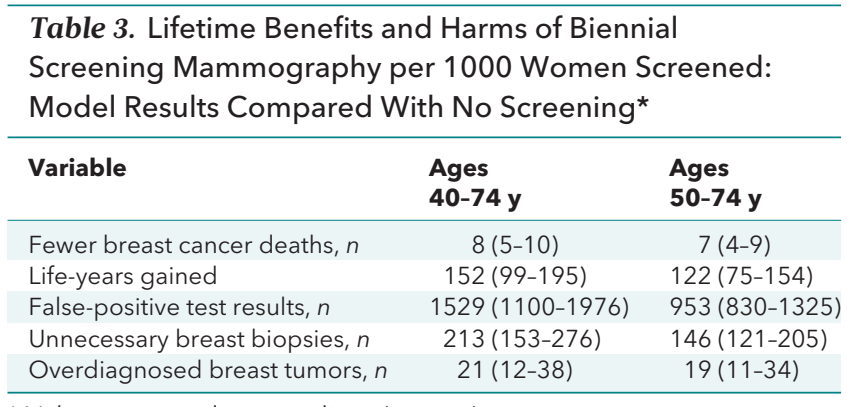

*Values reported are medians (ranges). ized trials that demonstrated the effectiveness of mammography in reducing breast cancer deaths in women aged 40 to 74 years, screening intervals ranged from 12 to 33 months $(2,3)$. There was no clear trend for greater benefit in trials of annual mammography, but other differences between the trials preclude certainty that no difference in benefit exists. Available observational evidence evaluating the effects of varying mammography intervals found no difference in the number of breast cancer deaths between women aged 50 years or older who were screened biennially versus annually $(2,3)$.

Regardless of the starting age for screening, the models consistently predict a small incremental increase in the number of breast cancer deaths averted when moving from biennial to annual mammography, but also a large increase in the number of harms (Table 4) $(7,8)$. The USPSTF concludes that for most women, biennial mammography screening provides the best overall balance of benefit and harms.

\section{When to Consider Stopping Screening}

Clinical trial data for women aged 70 to 74 years are inconclusive. In its 2009 recommendation (11), the USPSTF extended the recommendation for screening mammography to age 74 years based on the extrapolation that much of the benefit seen in women aged 60 to 69 years should continue in this age range, and modeling done at the time supported this assumption. Current CISNET models suggest that women aged 70 to 74 years with moderate to severe comorbid conditions that negatively affect their life expectancy are unlikely to benefit from mammography $(7,8,12)$. Moderate comorbid conditions include cardiovascular disease, paralysis, and diabetes. Severe comorbid conditions include (but are not limited to) AIDS, chronic obstructive pulmonary disease, liver disease, chronic renal failure, dementia, congestive heart failure, and combinations of moderate comorbid conditions, as well as myocardial infarction, ulcer, and rheumatologic disease (12).

\section{Screening in Women Aged 75 Years or Older}

The USPSTF found insufficient evidence to assess the balance of benefits and harms of screening mammography in women aged 75 years or older. CISNET models suggest that biennial mammography screening may potentially continue to offer a net benefit after age 74 years among those with no or low comorbidity $(7,8)$, but no randomized trials of screening included women in this age group $(2,3)$. 


\section{DBT as a Primary Screening Strategy}

The USPSTF found insufficient evidence to assess the balance of benefits and harms of DBT as a primary screening method for breast cancer.

\section{Background}

Evidence on DBT is limited; a single study on the test characteristics of DBT as a primary screening strategy for breast cancer met the inclusion criteria of the systematic evidence review (13).

\section{Potential Benefits}

From the limited data available, DBT seems to reduce recall rates (that is, follow-up for additional imaging or testing) and increase cancer detection rates compared with conventional digital mammography alone (13). However, current study designs cannot determine whether all of the additional cases of cancer detected would have become clinically significant (that is, the degree of overdiagnosis) or whether there is an incremental clinical benefit to detecting these cancers earlier than with conventional digital mammography. In addition, no studies of DBT looked at clinical outcomes, such as breast cancer morbidity or mortality or quality of life (13).

\section{Potential Harms}

As currently practiced in most settings, DBT exposes women to approximately twice the amount of radiation as conventional digital mammography (13). In 2013, the U.S. Food and Drug Administration approved a method to generate synthetic reconstruction of 2-dimensional images from 3-dimensional views, which reduces the total radiation dose associated with DBT. Although the extent to which this new software technology has been implemented in mammography screening centers is not precisely known, it is currently thought to be low. In women with abnormal findings, DBT may also increase the rate of breast biopsy compared with conventional digital mammography (13).

\section{Primary and Adjunctive Screening in Women With Dense Breasts}

The USPSTF found insufficient evidence to assess the balance of benefits and harms of adjunctive screening for breast cancer using breast ultrasonography, MRI, DBT, or other methods in women identified to have dense breasts on an otherwise negative screening mammogram.

\section{Epidemiology of Dense Breasts}

In the United States, the most commonly used classification system for breast density is the American College of Radiology's Breast Imaging Reporting and Data System (BI-RADS) 4-category scale $(a=$ the breasts are almost entirely fatty; $b=$ there are scattered areas of fibroglandular density; $c=$ the breasts are heterogeneously dense, which may obscure small masses; or $d=$ the breasts are extremely dense, which lowers the

www.annals.org

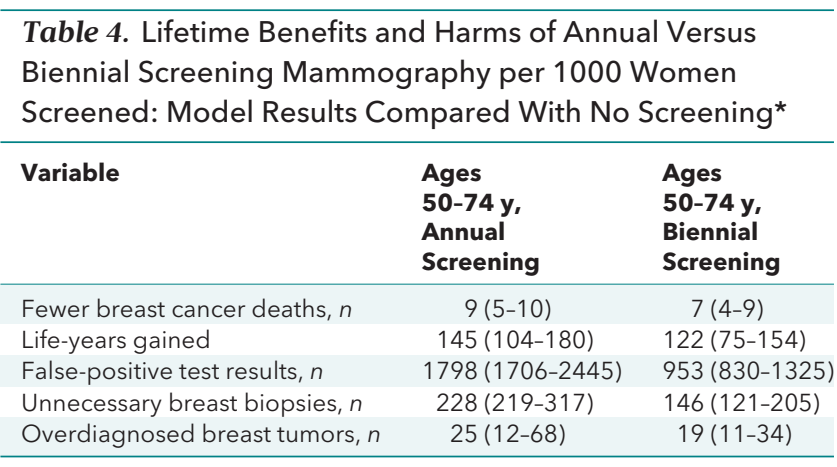

*Values reported are medians (ranges).

sensitivity of mammography). Data from the BCSC indicate that about 25 million women (about $43 \%$ ) aged 40 to 74 years are classified as having heterogeneously or extremely dense breasts. The proportion of women with dense breasts is highest among those aged 40 to 49 years and decreases with age (14).

Increased breast density is a risk factor for breast cancer. Data from the BCSC indicate that, compared with women with average breast density, women aged 40 to 49 years with heterogeneously or extremely dense breasts have a relative risk (RR) of 1.23 for developing invasive breast cancer. For women aged 50 to 64 years with heterogeneously or extremely dense breasts, the RR is 1.29 , and for women aged 65 to 74 years, it is 1.30 (7). However, women with dense breasts who develop breast cancer do not have an increased risk for dying from the disease, after adjustment for stage, treatment, method of detection, and other risk factors, according to data from the BCSC (15).

\section{Primary Screening Test Performance Characteristics}

Increased breast density reduces the sensitivity and specificity of mammography for detecting cancer. A BCSC study of more than 300000 women found that sensitivity decreased from $87 \%$ in the lowest density category to $63 \%$ in the highest, and specificity decreased from $96 \%$ to $90 \%$ as breast density increased (16).

A woman's BI-RADS breast density classification can be inconstant over time. Good-quality studies of U.S. radiologists demonstrate that major recategorization of sequential screening examinations (that is, from "dense" [c/d] to "nondense" [a/b] or vice versa) occurs in approximately $13 \%$ to $19 \%$ of women $(17,18)$. These studies excluded women taking hormone medications or those with other medical conditions that may have resulted in physiologic changes that would explain the difference in breast density classification observed between examinations. Reclassification of breast density status from year to year complicates women's assessment of their underlying breast cancer risk, as well as informed screening and care decisions.

\section{Primary Screening Frequency}

In 1 BCSC study, biennial screening mammography was associated with greater risk for advanced- 
stage cancer (stage IIB or greater) (odds ratio, $2.39[\mathrm{Cl}$, 1.06 to 3.39]) or a breast tumor larger than $20 \mathrm{~mm}$ (odds ratio, 2.39 [Cl, 1.37 to 3.18$]$ ) in women aged 40 to 49 years with extremely dense breasts (BI-RADS category d) compared with annual screening; this risk was not seen in women aged 50 to 74 years (19). No significant differences in lymph node involvement were observed in either age group. Information about morbidity or mortality end points is not available, so whether these women ultimately fared any differently in their clinical outcomes is not known $(17,19)$.

All women aged 40 to 74 years with increased breast density are at increased risk for a false-positive result, an unnecessary breast biopsy, or a falsenegative result compared with women with average breast density. Screening more frequently (that is, annually vs. biennially) further increases the probability that a woman will experience one of these screeningrelated harms. Data from the BCSC indicate that the cumulative probability that a woman aged 40 to 49 years with extremely dense breasts screened annually for a decade will receive a false-positive result is about $69 \%$, compared with about $21 \%$ for biennial screening. Similarly, unnecessary breast biopsy rates are $12 \%$ for annual screening versus $3 \%$ for biennial screening (17, 18).

\section{Adjunctive Screening}

Potential Benefits. Current evidence on adjunctive screening is very limited, but it suggests that for women identified to have dense breasts on an otherwise negative mammogram, ultrasonography or MRI will detect additional breast cancer but will also result in a higher number of false-positive results. Data on DBT in women with dense breasts are limited, but in the short term, DBT also detects additional breast cancer. Most of the additional cancer detected by these methods are invasive tumors rather than ductal carcinoma in situ (DCIS) $(17,18)$. A short-term increase in the number of cancer cases detected does not allow for the conclusion that adjunctive screening reduces treatment-related morbidity or breast cancer deaths or improves women's quality of life. Although adjunctive screening may detect more breast cancer, these cancers may fall into 1 of 3 categories: 1) those for which earlier detection leads to improved outcomes, 2) those that would have had the same outcome when detected later, or 3 ) those that are overdiagnosed and would not have caused a health problem during a woman's lifetime and may result in harms from unnecessary treatment. Existing data do not allow for estimation of the proportion of cancer that falls into each category; therefore, the benefits on health cannot be estimated.

Potential Harms. Most positive adjunctive breast cancer screening test results are false positive. Compared with mammography alone, adjunctive screening with ultrasonography or MRI seems to increase recall and biopsy rates. Data on the effects of DBT on recall and biopsy rates in women with dense breasts are too limited to draw conclusions $(17,18)$. The effects of DBT on overdiagnosis rates are unknown.
Current Practice. At the present time, 24 states require patient notification of breast density status when mammography is performed; in some states, legislation also includes language to be sent to women informing them that they should consider adjunctive screening (17). No clinical practice guidelines explicitly recommend adjunctive screening in women identified to have dense breasts on an otherwise negative screening mammogram (17).

\section{Assessment}

Increased breast density is very common. It is an independent risk factor for developing (but not dying of) breast cancer, and it reduces mammography's ability to find and accurately identify breast cancer. Many women will move between "dense" and "nondense" breast classifications with sequential screening mammograms, and these reclassifications are not primarily due to physiologic causes. More evidence is needed to better understand how the frequency of screening might affect important health outcomes in women with dense breasts. Overall, many important questions remain about the potential role of breast density in individualizing screening approaches, and the current evidence is insufficient to recommend a specific screening strategy for women with increased breast density.

\section{Other Approaches to Prevention}

The USPSTF has made recommendations about the use of medications to reduce women's risk for breast cancer, as well as risk assessment, genetic counseling, and genetic testing for BRCA1- or BRCA2related cancer (including breast cancer). These recommendations are available on the USPSTF Web site (www.uspreventiveservicestaskforce.org).

\section{Other Considerations \\ Research Needs and Gaps}

Trial data are too limited to directly inform the question of what the best screening strategy is for women or how clinicians can best tailor that strategy to the individual.

Overdiagnosis and resulting overtreatment of breast cancer that would otherwise not have become a threat to a woman's health during her lifetime is the most important harm associated with breast cancer screening. Because it is impossible to determine for any individual patient whether a diagnosed cancer will or will not progress, measurements of overdiagnosis are not straightforward but rather are indirectly quantified. Current estimates of the magnitude of overdiagnosis associated with mammography screening vary widely. Researchers in the field must work together to critically evaluate and ultimately agree on uniform definitions and standards to optimally measure and monitor overdiagnosis and overtreatment in breast cancer screening programs.

In addition, research is critically needed to identify ways to reduce the occurrence of overdiagnosis and subsequent overtreatment associated with breast can- 
cer screening. Ductal carcinoma in situ is an example of a breast lesion with the potential for high rates of overdiagnosis and overtreatment. Before the widespread use of screening mammography, 6 cases of DCIS per 100000 U.S. women per year were identified compared with 37 cases of DCIS per 100000 women per year after its introduction (5). When classified as cancer, DCIS now accounts for about 1 in 4 of all breast cancer diagnosed in a given year (20). However, its nomenclature has recently been the subject of debate, because by definition, DCIS is confined to the mammary ductallobular system and is incapable of metastasis (that is, it is noninvasive and thus lacks the classic characteristic of cancer) (21). Ductal carcinoma in situ may therefore be more appropriately classified as a risk factor for future development of cancer; the primary goal in its management is to reduce the incidence of new invasive carcinoma. The natural history of DCIS-particularly screendetected DCIS-is poorly understood. Although a substantial proportion of these lesions will not progress to invasive cancer (22), which women will and which will not develop such cancer cannot be predicted with certainty. As such, nearly all women diagnosed with DCIS receive treatment (generally either mastectomy or lumpectomy with or without radiation; a chemopreventive agent, such as tamoxifen, may also be offered) (23). The 20-year breast cancer mortality rate after treatment of DCIS is as low as 3\% (24); whether this is due to the effectiveness of the interventions or the fact that most DCIS cases being treated are essentially benign is a pressing research question. Research is needed to develop better prognostic indicators to distinguish nonprogressive or slowly progressive lesions from tumors that are likely to affect quality or length of life. Research is also needed to compare the long-term benefits and harms of immediate treatment versus observation or surveillance with delayed intervention in women with screen-detected DCIS.

Most of the available screening trials and highquality cohort studies were performed in Europe and predominately enrolled white women younger than age 70 years. Direct evidence about any differential effectiveness of breast cancer screening is lacking for important subgroups of women, such as African American women, who are at increased risk for dying of breast cancer, and older women, for whom balancing the potential benefits and harms of screening may become increasingly challenging with advancing age.

Newer technologies, such as DBT for primary screening or ultrasonography and MRI for adjunctive screening in women with dense breasts, are being increasingly used in the United States without clear evidence to demonstrate their effectiveness in improving important health outcomes. Such studies are necessary prerequisites for the appropriate incorporation of these methods into established screening programs.

Finally, a large proportion of women in the United States are classified as having dense breasts after screening mammography. Increased breast density is common in the general population; however, critical questions remain about how best to manage this con- dition and to support these women. Research to help improve the validity and reproducibility of serial BlRADS assessments would be useful if breast density is to be considered as a factor for personalized, riskbased approaches to breast cancer screening. In addition, long-term randomized trials or longitudinal cohort studies are needed that compare screening outcomes in women with dense breasts who are not otherwise at increased risk for breast cancer who receive adjunctive screening versus those who do not and report important outcomes, such as breast cancer stage at diagnosis, breast cancer recurrence rates, rates of overdiagnosis, and most importantly, breast cancer mortality.

\section{Discussion \\ Scope of Review}

The USPSTF commissioned a series of systematic evidence reviews in support of this recommendation. The first addressed the effectiveness of breast cancer screening in reducing breast cancer-specific and allcause mortality, as well as the incidence of advanced breast cancer and treatment-related morbidity. It also looked at the harms of breast cancer screening $(2-4,9)$. A second systematic review summarized the evidence about the test performance characteristics of DBT as a primary screening strategy (13). A third systematic review evaluated the evidence on adjunctive screening in women with increased breast density, including the accuracy and reproducibility of dense breast classification systems and the diagnostic test performance characteristics, benefits, and harms of adjunctive screening in women identified to have dense breasts on an otherwise negative screening mammogram $(17,18)$.

In addition to the systematic reviews of the evidence, the USPSTF commissioned a report from the CISNET Breast Cancer Working Group to provide information from comparative decision models on optimal starting and stopping ages and intervals for screening mammography, as well as how breast density, breast cancer risk, and comorbidity level affect the balance of benefit and harms of screening mammography $(7,8)$. A second decision analysis estimated the number of radiation-induced breast cancer cases and deaths associated with different screening mammography strategies over the course of a woman's lifetime $(25,26)$.

\section{Burden of Disease}

There are approximately 125 new cases of breast cancer and about 22 deaths per 100000 U.S. women each year. The mean age at diagnosis has remained unchanged at 64 years since the late 1970s (27). The median age at death is 68 years (1).

\section{Risk Factors: Additional Considerations}

About $5 \%$ to $10 \%$ of women who develop breast cancer have a mother or sister who also has breast cancer (2).

A few clinically significant factors are associated with high risk $(R R, \geq 4)$ for breast cancer (women with a $B R C A 1$ or BRCA2 gene mutation or other hereditary genetic syndromes or women with a history of high- 
dose radiation therapy to the chest at a young age, such as for treatment of Hodgkin lymphoma) (2). Women with these risk factors are not within the scope of this recommendation.

Race and ethnicity is a factor that has prompted concern because of a growing disparity in breast cancer mortality rates. Although white women have historically had higher incidence rates than African American women, incidence rates have come close to converging as of 2012 (128 vs. 124 cases per 100000 women per year, respectively) (28). More African American women die each year from breast cancer than white women (about 31 vs. 22 breast cancer deaths per 100000 women per year, respectively) (5). The reason for the difference in breast cancer mortality between white and African American women is not clear. It may be in part due to differences in biology-African American women are disproportionally affected by more aggressive and treatment-resistant forms of breast cancer (that is, cancer with adverse histologic features, such as poorly differentiated tumors and triple-negative phenotypes) (29, 30). Unfortunately, these types of cancer may be the least likely to be positively affected by screening programs, because they can grow so rapidly that they develop and spread entirely within the timespan between screening examinations. The difference in mortality rate may also be due to socioeconomic differences and health system failures. Multiple studies have shown an association between African American race and experiencing delays in receiving health care services for cancer, not receiving appropriate treatment, or not receiving treatment at all (31-33). African American women are also substantially underrepresented in RCTs of mammography screening. As such, there is no high-quality evidence to conclude that screening African American women more often or earlier than already recommended for the overall population of women would result in fewer breast cancer deaths or a greater net benefit.

\section{Accuracy of Screening Tests}

All available RCTs evaluating the effectiveness of breast cancer screening used film mammography. Despite a lack of direct evidence of effectiveness in reducing breast cancer deaths, conventional digital mammography has essentially replaced film mammography as the primary method for breast cancer screening in the United States. Conventional digital screening mammography has been shown to have about the same diagnostic accuracy as film, although digital screening seems to have comparatively higher sensitivity in women younger than age 50 years (34). Across all ages, screening mammography has a sensitivity of approximately $77 \%$ to $95 \%$ and a specificity of about $94 \%$ to 97\% (35).

Digital breast tomosynthesis is an emerging technology. One study on the test characteristics of DBT as a primary breast cancer screening strategy met the minimum inclusion criteria of the systematic evidence review (that is, the study needed to be conducted in an asymptomatic screening population, use a comprehen- sive reference standard that applied to both negative and positive test results, and have a minimum 1-year follow-up for negative results to ascertain interval breast cancer not identified by screening). As such, estimates of its test performance are subject to change with additional research. However, the positive predictive value of DBT (when used in conjunction with conventional digital mammography and calculated as the number of true positives [cancer] out of all positive examinations) ranges from $4.6 \%$ to $10.1 \%$ in U.S. studies (13).

Some information is available about the diagnostic test characteristics of adjunctive screening in women identified to have dense breasts on an otherwise negative screening mammogram. Handheld breast ultrasonography has the most evidence available (5 studies); its sensitivity to detect breast cancer ranges from $80 \%$ to $83 \%$, and its specificity ranges from $86 \%$ to $94 \%$, with a positive predictive value between $3 \%$ and $8 \%$. Three small studies of MRI in high-risk women found that its sensitivity to detect breast cancer ranged from $75 \%$ to $100 \%$, specificity ranged from $78 \%$ to $89 \%$, and positive predictive value ranged from $3 \%$ to $33 \%$, although the applicability of these studies to women in the general screening population is limited because of the highly selected population in these studies $(17,18)$.

\section{Effectiveness of Early Detection and Treatment Primary Screening With Conventional Mammography}

An updated meta-analysis by Nelson and colleagues of RCTs of screening mammography found similar RR reductions in breast cancer mortality by age group as the previous USPSTF evidence review. The combined RRs were $0.88(\mathrm{Cl}, 0.73$ to 1.003$)$ for women aged 39 to 49 years, $0.86(\mathrm{Cl}, 0.68$ to 0.97$)$ for women aged 50 to 59 years, $0.67(\mathrm{Cl}, 0.55$ to 0.91$)$ for women aged 60 to 69 years, and $0.80(\mathrm{Cl}, 0.51$ to 1.28) for women aged 70 to 74 years $(2,3)$.

None of the trials nor the combined meta-analysis demonstrated a difference in all-cause mortality with screening mammography (2).

Observational studies of screening mammography reported a wide range of breast cancer mortality reduction rates. Recent meta-analyses from the EUROSCREEN Working Group showed an approximate $25 \%$ to $31 \%$ relative reduction in breast cancer deaths in women aged 50 to 69 years who were invited to screening. In comparison, meta-analysis of RCTs that used an intention-to-treat analysis found a $19 \%$ to $22 \%$ breast cancer mortality reduction in women in the same age range $(2,3)$.

Updated decision models performed by CISNET yielded somewhat higher estimates in lifetime relative breast cancer mortality reductions with biennial mammography screening in women aged 50 to 74 years compared with previous analyses (median reduction, $25.8 \%$ vs. $21.5 \%$; range across models, $24.1 \%$ to $31.8 \%$ vs. $20.0 \%$ to $28.0 \%$, respectively). Since its previous analysis, CISNET has revised the inputs of each of its 6 models (for example, portraying distinct molecular sub- 
Table 5. Ten-Year Cumulative Probability (95\% Cl) of a False-Positive Test Result or Biopsy Recommendation From Annual or Biennial Mammography Screening Starting at Age 40 or 50 Years: Breast Cancer Surveillance Consortium Registry Data

\begin{tabular}{llllll}
\hline \multirow{2}{*}{ Variable } & \multicolumn{2}{c}{ Start at Age $\mathbf{4 0} \mathbf{y}$} & & \multicolumn{2}{c}{ Start at Age 50 y } \\
\cline { 2 - 3 } \cline { 5 - 6 } & Annual Screening & Biennial Screening & & Annual Screening & \multicolumn{1}{c}{ Biennial Screening } \\
\hline False-positive test result, \% & $61.3(59.4-63.1)$ & $41.6(40.6-42.5)$ & & $61.3(58.0-64.7)$ & $42.0(40.4-43.7)$ \\
False-positive biopsy recommendation, \% & $7.0(6.1-7.8)$ & $4.8(4.4-5.2)$ & & $9.4(7.4-11.5)$ & $6.4(5.6-7.2)$ \\
\hline
\end{tabular}

types and including digital mammography), which may account for some of the difference $(7,8)$. The updated estimate of the mortality benefit of mammography is also higher than that obtained via meta-analysis of randomized trials for a similar age group $(24.1 \%$ to $31.8 \%$ for women aged 50 to 74 years in decision models vs. $19 \%$ to $22 \%$ for women aged 50 to 69 years in RCTs) (2, 7). One reason for the discrepancy is the difference in the time horizon evaluated; whereas the meta-analysis looked at the impact of screening across a single decade, the decision models evaluated the impact of screening across an entire life span. It is also important to recognize that the decision models assumed perfect $(100 \%)$ adherence to screening, follow-up for abnormal findings, and treatment of screen-detected breast cancer for every patient. In addition, the models also assumed that all women receive the most effective, stagespecific treatments available for their breast cancer once it is detected by mammography. As such, the decision models represent an ideal, or the absolute maximum benefit, that a screening mammography program could achieve given no barriers to the delivery of health care services. In reality, the magnitude of benefit would be lower, given the real-world constraints of implementing a preventive service to such a large proportion of women in the United States.

In addition to mortality, other outcomes-such as quality of life or reduction in advanced-stage disease and any associated treatment-related morbidity-are also important to consider when evaluating the potential benefits of a screening program. From RCT evidence, meta-analysis indicated a reduced risk for advanced cancer with the use of screening mammography in women aged 50 years or older when "advanced disease" was defined by the most severe categories available (stages III and IV disease, tumor size $\geq 50 \mathrm{~mm}$, or $\geq 4$ positive lymph nodes) ( $R R, 0.62$ [Cl, 0.46 to 0.83$]$ ). A significant reduction in advanced disease was not observed with the use of screening mammography in women aged 40 to 49 years $(2,3)$. Data from observational evidence have shown mixed results; some studies showed an association between screening and reduced lymph node-positive disease or smaller tumor size (2), whereas others found no evidence of a change in advanced cancer rates as a result of mammography screening $(2,27)$.

The effect of screening mammography on associated adverse effects of treatment or their intensity is not clear from the literature. A meta-analysis of 5 RCTs showed that women randomly assigned to screening mammography were significantly more likely to have a

www.annals.org mastectomy (RR, $1.20[\mathrm{Cl}, 1.11$ to 1.30$])$ and surgical therapy (mastectomy and lumpectomy combined) (RR, $1.35[\mathrm{Cl}, 1.26$ to 1.44$])$ than women in the control groups (36). However, critics have noted that these trials do not reflect modern treatment standards and may therefore not represent current practices. Four caseseries included in the systematic evidence review compared breast cancer treatments in women who had previous mammography screening with those who did not and reported significantly more breast-conserving surgeries, fewer mastectomies, and less chemotherapy in women who had prior screening (2). However, all of these studies included women with DCIS in the denominator of screened women treated for cancer, leading to potential bias between the screened and nonscreened groups based on differences in how DCIS and invasive breast cancer are managed.

\section{Primary Screening With DBT}

No studies evaluated the effect of screening for breast cancer with DBT on important health outcomes, such as mortality, treatment-related morbidity, or quality of life (13).

Two case-series comparing conventional digital mammography versus DBT plus conventional digital mammography reported detection rates by cancer stage. One study $(n=29080)$ was conducted in the United States and the other $(n=12631)$ was conducted in Norway. Neither found significant differences in breast cancer size or node status at the time of diagnosis $(37,38)$.

Some evidence is available about the effect of DBT on recall rates for positive findings requiring additional evaluation. Nine studies compared findings from a single cohort of women undergoing 2 types of screening examinations or compared 2 screening cohorts of women (conventional digital mammography alone vs. combined with DBT). In the single study that met inclusion criteria for the systematic review, DBT combined with digital mammography was associated with a $0.6 \%$ reduction in immediate recall rates compared with digital mammography alone (recall rate, $3.6 \%$ and $4.2 \%$, respectively). Overall, across all available studies, DBT was associated with a median reduction in immediate recall rates of $1.7 \%$ (range, $0.6 \%$ to $7.2 \%$ ) (13).

\section{Adjunctive Screening in Women With Dense Breasts}

No studies evaluated the effects of adjunctive screening with any method in women with dense 
breasts on breast cancer rates, quality of life, or mortality $(17,18)$.

\section{Harms of Early Detection and Treatment Primary Screening With Conventional Mammography}

Screening mammography has several potential harms. The most common is a false-positive result, which can lead to psychological harms, as well as additional testing and invasive follow-up procedures. Studies show a fairly consistent association between a falsepositive screening mammogram and increased breast cancer-specific distress, anxiety, and apprehension, particularly in women who have an associated procedure, such as fine-needle aspiration or breast biopsy. These effects improve over time for most women $(2,4)$. Table 5 summarizes BCSC data on the cumulative probability of a woman (at varying starting ages and intervals) receiving at least 1 false-positive mammogram or a recommendation for what turns out to be a false-positive biopsy over a 10-year period (39).

The most serious harm of screening mammography is the diagnosis and treatment of breast cancer that would never have become a threat to a woman's health, or even apparent, during her lifetime (overdiagnosis and overtreatment). Overdiagnosis occurs when the breast tumor does not progress or when the woman dies of a competing cause of death before the breast cancer advances to the point of causing symptoms. Overdiagnosis is not the same as misdiagnosis. Misdiagnosis is when a cancer is incorrectly classified by an individual pathologist; overdiagnosis, on the other hand, happens when there is general agreement by pathologists on how to classify the cancer, but the tumor does not go on to behave as expected based on its appearance. It is not possible to directly observe for any individual woman whether she has or does not have an overdiagnosed tumor; it is only possible to indirectly estimate the frequency of overdiagnosis that may occur across a screened population. Researchers have used multiple data sources to attempt to quantify overdiagnosis rates associated with mammography screening, including RCTs, pathology and imaging studies, ecologic and cohort studies, and decision modeling. To additionally complicate matters, there is a lack of consensus concerning the optimal method for calculating the magnitude of overdiagnosis, and investigators differ in their approaches $(6,40)$. This has resulted in a wide range of estimates in the available literature $(0 \%$ to $54 \%)(2,4)$.

Of the available clinical trials, 3 RCTs in which there was no mammography screening of the control groups at the end of the study (Malmö Mammographic Screening Trial I and the Canadian National Breast Screening Study 1 and 2) provided the least-biased estimates, as they had the advantage of having comparable groups at baseline, adequate follow-up beyond the screening period to distinguish between earlier diagnosis and overdiagnosis, and clear distinction between which groups received screening and which did not (if screening was also provided to the control group, then over- diagnosis could also occur in this population) (6). These older trials likely underestimate the actual magnitude of overdiagnosis associated with modern screening mammography programs, given the increasing sensitivity of newer technologies, but together, they suggest that over a 10 -year period, approximately $19 \%$ of breast cancers are overdiagnosed $(2,4)$. CISNET decision models also investigated the degree of overdiagnosis likely to result from a screening mammography program. The 6 decision models reported a wide range of estimates of the magnitude of overdiagnosis associated with screening mammography $(1.4 \%$ to $24.9 \%$ of invasive cancer and $30.5 \%$ to $84.5 \%$ of DCIS, depending on the screening strategy) $(7,8)$. Assumptions in several of the models may have increased the likelihood of underestimating the true burden of overdiagnosis associated with screening mammography. Most importantly, 4 of the 6 models assumed that all diagnosed invasive cancer can progress to lethality; only 1 (model W) allowed for the possibility of cancer with "limited malignant potential," whereby the tumor stops progressing at an early invasive stage. In addition, 1 of the models omitted DCIS.

Recurrent radiation exposure from a lifetime program of mammography screening may slightly increase the risk for breast cancer, although no empirical studies have directly measured this effect. Simulation models performed in support of this scientific report estimate that the mean lifetime attributable risk (LAR) of radiation-induced breast cancer from biennial screening mammography in women aged 50 to 74 years is 3 cases per 10000 women screened. The mean LAR of breast cancer death is 0.5 deaths per 10000 women screened. If biennial screening begins at age 40 instead of 50 years, the mean LAR of developing breast cancer increases to 4 cases per 10000 women screened, and the number of breast cancer deaths increases to about 1 per 10000 women screened (25, 26). Of note, women with large breasts, who may require extra views-and thus higher radiation doses-for complete mammography examination, seem to be at increased risk for radiation-induced breast cancer or breast cancer death. Based on information from the Digital Mammography Imaging Screening Trial (which compared the test characteristics of film vs. digital mammography) (41), as no representative populationbased data are available, an estimated $5 \%$ to $6 \%$ of U.S. women will require additional views during screening for complete breast examination. For biennial screening in women aged 50 to 74 years, the mean LAR of developing breast cancer is an estimated 6 versus 2 cases per 10000 screened women with and without large breasts, respectively; the mean LAR of breast cancer death is 1 versus 0.4 deaths per 10000 screened women with and without large breasts, respectively (25, 26).

\section{Primary Screening With DBT}

Currently, DBT is most frequently performed in combination with conventional digital mammography; 
this practice essentially doubles the resulting radiation exposure to the patient. The U.S. Food and Drug Administration has approved a method to generate synthetic reconstructions of 2-dimensional images from 3-dimensional views, which reduces the total radiation dose emitted. However, study data on the performance of DBT in isolation (that is, with synthetic reconstruction of 2-dimensional views) is limited to 1 mammography reading study that compared sensitivity and specificity and 1 prospective clinical trial (42), and the method is not yet thought to be in widespread clinical use.

Limited evidence suggests that DBT may slightly increase the risk for breast biopsy compared with conventional digital mammography. In 4 U.S. studies of DBT that reported breast biopsy rates, 3 noted higher rates in the combined DBT and conventional digital mammography group compared with conventional digital mammography alone (median difference, $0.2 \%$ [range, $-0.1 \%$ to $0.4 \%]$ ) (13).

\section{Adjunctive Screening in Women With Dense Breasts}

Although evidence is limited, the use of adjunctive screening in women with increased breast density via alternative technologies, such as handheld ultrasonography or MRI, generally seems to increase recall and breast biopsy rates compared with standard screening mammography alone $(17,18)$. A single good-quality U.S. study that evaluated the use of adjunctive handheld ultrasonography and MRI found that the recall rate for handheld ultrasonography after a negative mammogram was about $14 \%$ compared with $11 \%$ for primary screening mammography alone. In women who received adjunctive screening with MRI after a negative mammogram and negative ultrasound, the recall rate was $23 \%$ (43).

\section{Estimate of Magnitude of Net Benefit}

For women who are not known to be at increased risk for breast cancer, the value of screening mammography increases with age, with the greatest benefit occurring from ages 50 to 74 years. In particular, women aged 60 to 69 years are the most likely to avoid a breast cancer death. Screening women every 2 years provides the best balance of benefit and harms. For women aged 40 to 49 years, the potential benefit is smaller, and the risk for harms is proportionally greater. However, the potential outcomes that need to be considered are not identical, and individual women may differ in how they prioritize them. The small probability that a woman may avoid a breast cancer death must be weighed against the more likely scenario that she may have a false-positive result and possible unnecessary follow-up testing (including invasive testing); a falsenegative result, with false reassurance or delayed diagnosis; or most critically, diagnosis and treatment of cancer that would otherwise not have threatened her health or even come to her attention. Women who value the possible breast cancer mortality benefit more than they value avoiding the harms can make an informed decision to begin screening. For women aged 75 years or older, evidence is very limited concerning the benefits and harms of screening mammography. However, since the mortality benefits of screening mammography (as with almost any cancer screening test) generally take years to accrue but many of the harms can be experienced immediately, women with limited life expectancy or severe comorbid conditions are unlikely to benefit.

Digital breast tomosynthesis is an emerging technology for breast cancer screening. Preliminary evidence suggests that it can reduce recall rates for falsepositive results and detect more cancer compared with conventional digital mammography. However, it may increase breast biopsy rates, and as currently practiced in most settings, DBT exposes women to more radiation than conventional 2-dimensional mammography. It is not clear whether all of the extra cancer cases detected by DBT actually represent a benefit (that is, cancer that is clinically significant rather than overdiagnosis, and of any additional benefit compared with detection by conventional digital mammography at the next scheduled examination). Most importantly, no studies assessed the effect of DBT on important health outcomes for women, such as quality of life, morbidity, or mortality. Finally, increased breast density is a common condition that imparts some increased risk for breast cancer, and it reduces the test performance characteristics of mammography as well. Current evidence on the use of adjunctive screening in women with increased breast density is not sufficient to recommend a specific screening strategy. These are important areas for future research.

\section{Update of Previous USPSTF RECOMMENDATION}

This recommendation updates the 2009 USPSTF recommendation on breast cancer screening with an assessment of the most current available scientific evidence for mammography screening. In addition, this update also provides additional clarity on what is meant by the " $C$ " recommendation for women aged 40 to 49 years. $A$ " $C$ " recommendation is not a recommendation against mammography screening in this age group; it signifies moderate certainty of a net benefit for screening that is small in magnitude. $A$ " $C$ " recommendation emphasizes that the decision to screen should be an individual one, made after a woman weighs the potential benefit against the possible harms. This recommendation also notes that women aged 40 to 49 years with a first-degree relative (parent, child, or sibling) with breast cancer may potentially benefit more than average-risk women in this age group from beginning screening mammography before age 50 years.

The USPSTF did not update its recommendation on whether there are additional, incremental benefits or harms associated with the use of MRI or digital mammography instead of film mammography in women who are not at increased risk for breast cancer, given that digital mammography has now essentially replaced film mammography as the primary breast can- 
cer screening method in the United States. The USPSTF did not update its 2009 recommendation on teaching breast self-examination; the USPSTF supports all patients being aware of changes in their bodies and discussing these changes with clinicians. The USPSTF also did not update its recommendation on the additional potential benefit of clinical breast examination.

This recommendation examines the evidence of the effectiveness of an emerging technology-DBT-as a primary screening strategy. It also evaluates the effectiveness of adjunctive screening using ultrasonography, MRI, DBT, or other methods in women identified to have dense breasts on an otherwise negative mammogram.

The scope of the evidence review supporting this recommendation statement was determined after public comment on the draft research plan.

\section{Response to Public Comment}

A draft recommendation statement was posted for public comment on the USPSTF Web site from 21 April through 18 May 2015. In response to the comments received, the USPSTF clarified certain terminology (for example, DBT and misdiagnosis vs. overdiagnosis), updated or added references (for example, those related to the long-term outcomes of DCIS), and provided additional context around the potential risks of radiation exposure due to mammography screening. Additional discussion of the public comments is below.

\section{Benefits of Contemporary Screening Mammography}

Some commenters thought that the USPSTF did not consider the available observational evidence on the effectiveness of mammography screening, and that the USPSTF's assessment is therefore based on outdated information, resulting in an underestimate of the current benefits of screening. This is incorrect. The systematic evidence review, which served as the foundation for this recommendation statement, encompassed both RCTs and nonexperimental studies of mammography screening. Nearly 200 observational studies, including 83 that specifically evaluated the benefits of screening mammography, were captured in the review (2). The USPSTF agrees that more contemporary evidence on breast cancer screening is important to consider given the age of the available RCTs, and it reviewed both the recent Pan-Canadian (44) and the Swedish Mammography Screening in Young Women (45) cohort studies. As noted in the Discussion section, RCTs of mammography screening have generally found smaller relative reductions in breast cancer mortality than observational studies, although estimates from the latter vary. For example, meta-analyses of mammography screening in women aged 50 to 69 years showed RRs of breast cancer death ranging from 0.78 to 0.81 in RCTs compared with about 0.69 to 0.75 in observational studies (2). There may be several reasons for this difference in the magnitude of benefit. Improvements in mammography technology may be translating into an increased mortality benefit, so that more recent observational evidence demonstrates a real difference in the efficacy of modern-day screening compared with that of the older randomized trials. However, known and important methodological limitations of observational studies, such as selection bias, lead and length time bias, and residual or unknown bias and confounding, are likely explanations for some or even all of the difference observed. In the absence of a modern-day clinical trial, it is impossible to know with certainty the precise magnitude of benefit of current screening mammography programs. As such, the USPSTF believes it is most appropriate to consider all of the evidence available (given that it meets prespecified and transparent quality standards, such as those delineated in its commissioned systematic evidence review) (2), while acknowledging that uncertainty remains about the exact size of the anticipated benefit in either direction.

\section{Screening Mammography in Women Aged 40 to 49 Years}

Some commenters incorrectly believed that the " $\mathrm{C}$ " recommendation for women aged 40 to 49 years represented a change from what the USPSTF had recommended in the past. Others thought that the " $\mathrm{C}$ " recommendation meant that the USPSTF was recommending against screening in this group of women. As noted previously, the recommendation for women aged 40 to 49 years was also a "C" in 2009 (meaning that the USPSTF had concluded that it had moderate certainty of a small net benefit to mammography screening in this population). This update clarifies the language around what the " $\mathrm{C}$ " recommendation means. It is not a recommendation against screening but a statement that the decision to undergo screening mammography for women in their 40s should be an informed, individual one, after she weighs the potential benefit against the potential harms.

\section{Screening Mammography in Women Aged 70 to 74 Years or Older}

Several commenters believed that the USPSTF was inconsistent in extending a " $\mathrm{B}$ " recommendation to women aged 70 to 74 years but assigning an "I" statement to women aged 75 years or older. These commenters noted that although the USPSTF had explained that trial data were inconclusive for women in their early 70s, modeling data could support extending the benefit seen in younger women to this age range. However, the USPSTF had stated that modeling data were not sufficient to establish the benefits of mammography screening in women aged 75 years or older. This is an apparent, though not real, contradiction. Two RCTs of mammography screening included participants aged 70 to 74 years. A meta-analysis performed to support the 2002 USPSTF recommendations found that the $\mathrm{RR}$ reduction of breast cancer death in screened women aged 65 to 74 years was $0.78(\mathrm{Cl}, 0.62$ to 0.99$)$ (35). In 2009 and 2014, the meta-analyses were restricted to women aged 70 to 74 years. In the most recent update, the meta-analysis showed an RR reduc- 
tion of $0.80(\mathrm{Cl}, 0.51$ to 1.28$)(2)$, and the $\mathrm{Cl}$ widened and crossed 1.0 because of the small number of absolute participants included in the analysis. The USPSTF considered the trial data, which were suggestive but not definitive, showing a benefit to mammography in this age group within the context of the modeling data, which also suggested an absolute breast cancer mortality benefit (with reasonable additional harms) with the extension of screening to this age group. In women aged 75 years or older, however, direct evidence about the benefits of screening mammography does not exist; no clinical trials of screening include this population (2). Therefore, in this case, the USPSTF's assessment could not rest on a foundation of empirical data augmented with supplemental information, but would have to be entirely based on decision modeling. The USPSTF does not use evidence from models alone to establish that a clinical preventive service is effective or harmful; rather, it uses modeling as an important extension that builds on observed evidence.

\section{Definition of Overdiagnosis}

The USPSTF received several comments about how overdiagnosis was conceptualized in the recommendation statement. Commenters stressed that it is impossible to directly measure overdiagnosis, and that there are multiple methods for estimating its frequency but no scientific consensus on the optimal approach. The USPSTF agrees, and it discusses these issues in several places throughout the recommendation. Commenters emphasized that because it is not possible to know whether any individual screen-detected cancer would have been clinically detected or gone on to cause health problems in the absence of screening, clinicians treat all detected breast cancer as potentially lethal; again, the USPSTF concurs. Commenters disagreed about the degree of overdiagnosis that is likely to occur with the use of mammography screening, and the USPSTF received requests to emphasize percentages that are both higher and lower than the 19\% estimate derived from the trials that did not have screening in their control groups at the end of the study. For the sake of transparency, the USPSTF has provided the full range of overdiagnosis estimates available in the literature as identified in its commissioned systematic evidence review, as well as specific estimates from observational studies, randomized trials, and modeling studies, with the clear caveat that ongoing uncertainty remains. This uncertainty is not about the fundamental existence of this critical harm of mammography screening (46), as a few commenters suggested; rather, it is about the precise magnitude of the problem. The USPSTF notes that continuing research is essential to advance understanding of the magnitude of overdiagnosis and how to distinguish overdiagnosed cancer from cancer that is likely to progress, as well as to reduce its occurrence.

\section{$D B T$}

Some commenters thought the USPSTF overlooked evidence in its review of the efficacy of DBT.
Commenters noted that studies have shown that DBT increases the detection of breast cancer and reduces recalls and false-positive results. The USPSTF commissioned a systematic evidence review on DBT as a primary screening method for breast cancer (13). The original search identified 945 studies, of which only 13 were identified as specifically relevant to the topic at hand. A bridge search performed during the public comment period to keep the review as temporally current as possible identified another 79 studies, 5 of which directly addressed the test characteristics of DBT. Despite this expansive and meticulous search, a single study met the prespecified inclusion criteria (studies must be conducted in a screening population of asymptomatic women aged 40 years or older and must evaluate test performance characteristics against a comprehensive reference standard that is applied to both negative and positive test results). A "comprehensive reference standard" means that further imaging and/or biopsy is performed for positive results, and there is a minimum of 1 year of clinical follow-up for negative results in order to accurately assess the interval breast cancer rate (that is, the number of cancers that appear in between screening periods, which allows for the calculation of false-negative results). Even though no other studies met these inclusion criteria, the USPSTF still carefully reviewed the data from all of the studies identified as directly relevant to the use of DBT for primary breast cancer screening, including Friedewald and colleagues' 2014 study that evaluated the performance of DBT in combination with conventional digital mammography (47). As already noted in the Clinical Considerations section, the USPSTF agrees that preliminary evidence suggests that DBT can reduce recall rates for false-positive results (median reduction, $1.7 \%$ [range in the systematic review, $0.6 \%$ to $7.2 \%]$ ) (13). The USPSTF also agrees that DBT appears to increase the cancer detection rate compared with conventional digital mammography alone. However, the rate of overdiagnosis associated with DBT is unknown; it is also unknown if there is an incremental benefit to finding these cancers earlier than with conventional digital mammography. The USPSTF recommends continued research around this emerging technology.

\section{Comparison of the USPSTF's Recommendations With Those of Other Organizations}

Some commenters noted that the USPSTF recommendations on breast cancer screening do not align with those of other organizations, such as the American College of Radiology (48) or the American Congress of Obstetricians and Gynecologists (49)-both of whom recommend annual mammography screening beginning at age 40 years-and were concerned that the lack of conformity may be confusing to clinicians and patients. While there are some differences, it is worth emphasizing the notable similarities in the approach of many major guidelines, including those between the USPSTF's recommendations and the recently released 
recommendations from the American Cancer Society (50). The USPSTF stands in agreement with most organizations that mammography screening can reduce deaths due to breast cancer in women in their 40s. Based on the evidence, the USPSTF found that there is a net benefit for women to initiating screening in their $40 \mathrm{~s}$, and that the size of the net benefit is smaller than that for older women; it therefore concludes that the decision to begin screening should be an individual one. The American Cancer Society, along with many other groups, including the American College of Physicians (51), the American Academy of Family Physicians (52), the 2013 Well-Woman Task Force assembled by the American Congress of Obstetricians and Gynecologists $(53,54)$, and the Canadian Task Force on Preventive Health Care (55), also recommends individualized decision making for women in their 40s on whether and when to initiate mammography screening. The USPSTF clarified that the risk for breast cancer increases with age (so a 48-year-old woman's potential to benefit may be more similar to a 50- rather than a 40-year-old woman), and that women may wish to factor this information into their decision making as they consider whether and when to initiate screening in their 40s. Notably, the USPSTF is in agreement with all other major professional organizations about the importance of regular mammography screening for women aged 50 to 74 years. The USPSTF found that most of the benefit of mammography screening is realized with biennial screening. The newly released recommendations from the American Cancer Society also support biennial screening, at intervals of 1 to 2 years in women in their 50s, 60s, and 70s (50). National breast cancer screening programs in the United Kingdom, the Netherlands, Switzerland, Poland, Norway, Luxembourg, Germany, Finland, Denmark, and Belgium offer mammography screening every 2 to 3 years for women aged 50 up to 74 years (56). Many European countries adhere to recommendations from the International Agency for Research on Cancer, which recently updated its guidelines to promote screening starting at age 50 years (57).

\section{Insurance Coverage and Access to Mammography}

Some commenters expressed concern that a " $\mathrm{C}$ " recommendation from the USPSTF might create a financial barrier for some women in their 40s who wish to undergo mammography screening after making an informed decision. These commenters asked the USPSTF to change its recommendation to a " $B$ " to guarantee insurance coverage for these women. The Affordable Care Act mandates that persons with private health plans receive coverage without copayment or coinsurance for preventive services that have a USPSTF "A" or "B" recommendation (58).

Recommendations from the USPSTF are based on its interpretation of the science regarding the potential benefits and harms of a preventive service; a " $\mathrm{C}$ " recommendation means that the USPSTF has concluded that there is at least moderate certainty of a small net benefit to the service, whereas a " $\mathrm{B}$ " recommendation means that there is either high certainty that the net benefit is moderate or moderate certainty that the net benefit is moderate to substantial. The USPSTF found in 2009 and again in 2016 that there is moderate certainty of a small net benefit to starting breast cancer screening before age 50 years because of the balance of benefit and harms outlined in this recommendation statement. The USPSTF could not give a "B" recommendation for screening in women aged 40 to 49 years because the science does not support moderate or high certainty of moderate or substantial net benefit in this age group. The role of the USPSTF is to assess the scientific evidence for preventive services; it is not within its mandate to reinterpret the science and inflate the net benefit of mammography screening solely to ensure insurance coverage of the service for women with private insurance plans (59). The USPSTF recommends that women in their 40s who understand the potential harms of mammography screening but place a higher value on the potential benefit have the option to begin screening. The USPSTF appreciates that, in the absence of full or partial insurance coverage, fewer women may make that choice, but those determinations are made by payers and legislators. Private and public payers have the option of providing coverage (as most do), and legislators have the option of requiring coverage (as they have done in the past and again in the 2016 Consolidated Appropriations Act) (60).

Some commenters highlighted the importance of coverage without additional payment for women with Medicare or Medicaid. Coverage in these plans does not reflect USPSTF recommendations and is decided separately by the Centers for Medicare \& Medicaid Services (for Medicare) and by individual states (for Medicaid).

Commenters also expressed concern that mammography is currently underutilized in the United States in some settings (for example, rates of recent use among women aged 50 to 74 years do not meet the Healthy People 2020 target [61]) and that any recommendation other than annual mammography screening beginning at age 40 years might "drive complacency" among women and cause them to delay needed attention to their health. The most recent trend data from the National Committee for Quality Assurance suggest that mammography screening rates have increased among women in all insurance categories since the USPSTF's 2009 recommendation (62). As with most effective medical interventions, there is the potential for underuse, appropriate use, and overuse of screening mammography in the general population. The USPSTF agrees that effective patient, provider, and systemslevel interventions should be employed to increase the appropriate use of screening mammography among women who are most likely to benefit from it, and it feels strongly that women need accurate information about potential benefits and harms so they can make the best decision for themselves.

Disclaimer: Recommendations made by the USPSTF are independent of the U.S. government. They should not be con- 
strued as an official position of the Agency for Healthcare Research and Quality or the U.S. Department of Health and Human Services.

Financial Support: The USPSTF is an independent, voluntary body. The U.S. Congress mandates that the Agency for Healthcare Research and Quality support the operations of the USPSTF.

Disclosures: Dr. Phillips disclosed that he has received past grants and contracts from $\mathrm{NCl}$ and $\mathrm{CDC}$ for research on patterns of use of screening tests for breast and other cancers in primary care. Dr. owens discloses that he has received travel reimbursement from the USPSTF. Authors followed the policy regarding conflicts of interest described at www.uspreventive servicestaskforce.org/Page/Name/methods-and-processes. Forms can be viewed at www.acponline.org/authors/icmje/ ConflictOfInterestForms.do?msNum=M15-2886.

\section{References}

1. SEER Stat Factsheets: Breast Cancer. Bethesda, MD: National Cancer Institute; 2015. Accessed at http://seer.cancer.gov/statfacts/html /breast.html on 13 April 2015.

2. Nelson HD, Cantor A, Humphrey L, Fu R, Pappas M, Daeges M, et al. Screening for Breast Cancer: A Systematic Review to Update the 2009 U.S. Preventive Services Task Force Recommendation. Evidence Synthesis No. 124. AHRQ Publication No. 14-05201-EF-1. Rockville, MD: Agency for Healthcare Research and Quality; 2015.

3. Nelson HD, Fu R, Cantor A, Pappas M, Daeges M, Humphrey L. Effectiveness of breast cancer screening: systematic review and meta-analysis to update the 2009 U.S. Preventive Services Task Force recommendation. Ann Intern Med. 2016. [Epub ahead of print]. doi: 10.7326/M15-0969

4. Nelson HD, Pappas M, Cantor A, Griffin J, Daeges M, Humphrey L. Harms of breast cancer screening: systematic review to update the 2009 U.S. Preventive Services Task Force recommendation. Ann Intern Med. 2016. [Epub ahead of print]. doi: 10.7326/M15-0970

5. Howlader N, Noone AM, Krapcho M, Garshell J, Miller D, Altekruse SF, et al, eds. SEER Cancer Statistics Review, 1975-2011. Bethesda, MD: National Cancer Institute; 2014. Accessed at http: //seer.cancer.gov/csr/1975_2011/ on 13 April 2015.

6. Marmot MG, Altman DG, Cameron DA, Dewar JA, Thompson SG, Wilcox $\mathrm{M}$. The benefits and harms of breast cancer screening: an independent review. $\mathrm{Br} J$ Cancer. 2013;108:2205-40. [PMID: 23744281] doi:10.1038/bjc.2013.177

7. Mandelblatt J, Cronin K, de Koning H, Miglioretti DL, Schechter C, Stout N. Collaborative Modeling of U.S. Breast Cancer Screening Strategies. AHRQ Publication No. 14-05201-EF-4. Rockville, MD: Agency for Healthcare Research and Quality; 2015.

8. Mandelblatt JS, Stout NK, Schechter CB, van den Broek JJ, Miglioretti DL, Krapcho M, et al. Collaborative modeling of the benefits and harms associated with different U.S. breast cancer screening strategies. Ann Intern Med. 2016. [Epub ahead of print]. doi: 10.7326/M15-1536

9. Nelson HD, O'Meara ES, Kerlikowske K, Balch S, Miglioretti D. Factors associated with rates of false-positive and false-negative results from digital mammography screening: an analysis of registry data. Ann Intern Med. 2016. [Epub ahead of print]. doi: 10.7326 /M15-0971

10. Nelson HD, Smith ME, Griffin JC, Fu R. Use of medications to reduce risk for primary breast cancer: a systematic review for the U.S. Preventive Services Task Force. Ann Intern Med. 2013;158:604-14. [PMID: 23588749] doi:10.7326/0003-4819-158-8-201304160-00005 11. U.S. Preventive Services Task Force. Screening for breast cancer: U.S. Preventive Services Task Force recommendation statement. Ann
Intern Med. 2009;151:716-26. [PMID: 19920272] doi:10.7326/0003 -4819-151-10-200911170-00008

12. Lansdorp-Vogelaar I, Gulati R, Mariotto AB, Schechter $C B$, de Carvalho TM, Knudsen $A B$, et al. Personalizing age of cancer screening cessation based on comorbid conditions: model estimates of harms and benefits. Ann Intern Med. 2014;161:104-12. [PMID: 25023249] doi:10.7326/M13-2867

13. Melnikow J, Fenton JJ, Miglioretti D, Whitlock EP, Weyrich MS Screening for Breast Cancer With Digital Breast Tomosynthesis. Evidence Synthesis No. 125. AHRQ Publication No. 14-05201-EF-2. Rockville, MD: Agency for Healthcare Research and Quality; 2015.

14. Sprague BL, Gangnon RE, Burt V, Trentham-Dietz A, Hampton JM, Wellman RD, et al. Prevalence of mammographically dense breasts in the United States. J Natl Cancer Inst. 2014;106. [PMID: 25217577] doi:10.1093/jnci/dju255

15. Gierach GL, Ichikawa L, Kerlikowske K, Brinton LA, Farhat GN Vacek PM, et al. Relationship between mammographic density and breast cancer death in the Breast Cancer Surveillance Consortium. J Natl Cancer Inst. 2012;104:1218-27. [PMID: 22911616] doi:10 .1093/jnci/djs327

16. Carney PA, Miglioretti DL, Yankaskas BC, Kerlikowske K, Rosenberg R, Rutter CM, et al. Individual and combined effects of age, breast density, and hormone replacement therapy use on the accuracy of screening mammography. Ann Intern Med. 2003;138:16875. [PMID: 12558355]

17. Melnikow J, Fenton JJ, Whitlock EP, Miglioretti DL, Weyrich MS, Thompson JH, et al. Supplemental Screening for Breast Cancer in Women With Dense Breasts: A Systematic Review for the U.S. Preventive Service Task Force. Evidence Synthesis No. 126. AHRQ Publication No. 14-05201-EF-3. Rockville, MD: Agency for Healthcare Research and Quality; 2015.

18. Melnikow J, Fenton JJ, Whitlock EP, Miglioretti DL, Weyrich MS, Thompson JH, et al. Supplemental screening for breast cancer in women with dense breasts: a systematic review for the U.S. Preventive Services Task Force. Ann Intern Med. 2016. [Epub ahead of print]. doi: 10.7326/M15-1789

19. Kerlikowske K, Zhu W, Hubbard RA, Geller B, Dittus K, Braithwaite $D$, et al; Breast Cancer Surveillance Consortium. Outcomes of screening mammography by frequency, breast density, and postmenopausal hormone therapy. JAMA Intern Med. 2013;173:807-16. [PMID: 23552817] doi:10.1001/jamainternmed.2013.307

20. Siegel R, Ma J, Zou Z, Jemal A. Cancer statistics, 2014. CA Cancer J Clin. 2014;64:9-29. [PMID: 24399786] doi:10.3322/caac.21208 21. Esserman LJ, Thompson IM Jr, Reid B. Overdiagnosis and overtreatment in cancer: an opportunity for improvement. JAMA. 2013; 310:797-8. [PMID: 23896967]

22. Wickerham DL, Julian TB. Ductal carcinoma in situ: a rose by any other name [Editorial]. J Natl Cancer Inst. 2013;105:1521-2. [PMID: 24068770] doi:10.1093/jnci/djt268

23. Baxter NN, Virnig BA, Durham SB, Tuttle TM. Trends in the treatment of ductal carcinoma in situ of the breast. J Natl Cancer Inst. 2004;96:443-8. [PMID: 15026469]

24. Narod SA, lqbal J, Giannakeas V, Sopik V, Sun P. Breast cancer mortality after a diagnosis of ductal carcinoma in situ. JAMA Oncol. 2015;1:888-96. [PMID: 26291673] doi:10.1001/jamaoncol.2015 .2510

25. Miglioretti DL, Lange J, van Ravesteyn N, van den Broek JJ, Lee $\mathrm{Cl}$, Melnikow J, et al. Radiation-Induced Breast Cancer and Breast Cancer Death From Mammography Screening [Abstract]. AHRQ Publication No. 14-05201-EF-5. Rockville, MD: Agency for Healthcare Research and Quality; 2015.

26. Miglioretti DL, Lange J, van den Broek JJ, Lee Cl, van Ravesteyn NT, Ritley $D$, et al. Radiation-induced breast cancer incidence and mortality from digital mammography screening. A modeling study. Ann Intern Med. 2016. [Epub ahead of print]. doi: 10.7326/M15 $-1241$

27. Welch HG, Gorski DH, Albertsen PC. Trends in metastatic breast and prostate cancer-lessons in cancer dynamics. N Engl J Med. 2015;373:1685-7. [PMID: 26510017] doi:10.1056/NEJMp1510443 
28. DeSantis CE, Fedewa SA, Goding Sauer A, Kramer JL, Smith RA, Jemal A. Breast cancer statistics, 2015: Convergence of incidence rates between black and white women. CA Cancer J Clin. 2015. [PMID: 26513636] doi:10.3322/caac.21320

29. Bauer KR, Brown M, Cress RD, Parise CA, Caggiano V. Descriptive analysis of estrogen receptor (ER)-negative, progesterone receptor (PR)negative, and HER2-negative invasive breast cancer, the so-called triplenegative phenotype: a population-based study from the California cancer Registry. Cancer. 2007;109:1721-8. [PMID: 17387718]

30. Carey LA, Perou CM, Livasy CA, Dressler LG, Cowan D, Conway $\mathrm{K}$, et al. Race, breast cancer subtypes, and survival in the Carolina Breast Cancer Study. JAMA. 2006;295:2492-502. [PMID: 16757721] 31. Brawley OW. Is race really a negative prognostic factor for cancer? [Editorial]. J Natl Cancer Inst. 2009;101:970-1. [PMID: 19567421] doi:10.1093/jnci/djp185

32. Griggs JJ, Culakova E, Sorbero ME, Poniewierski MS, Wolff DA, Crawford J, et al. Social and racial differences in selection of breast cancer adjuvant chemotherapy regimens. J Clin Oncol. 2007;25: 2522-7. [PMID: 17577029]

33. Roetzheim RG, Pal N, Tennant C, Voti L, Ayanian JZ, Schwabe A, et al. Effects of health insurance and race on early detection of cancer. J Natl Cancer Inst. 1999;91:1409-15. [PMID: 10451447]

34. Souza FH, Wendland EM, Rosa MI, Polanczyk CA. Is full-field digital mammography more accurate than screen-film mammography in overall population screening? A systematic review and metaanalysis. Breast. 2013;22:217-24. [PMID: 23489759] doi:10.1016/j .breast.2013.02.013

35. Humphrey LL, Helfand M, Chan BK, Woolf SH. Breast cancer screening: a summary of the evidence for the U.S. Preventive Services Task Force. Ann Intern Med. 2002;137:347-60. [PMID: 12204020]

36. Gøtzsche PC, Jørgensen KJ. Screening for breast cancer with mammography. Cochrane Database Syst Rev. 2013;6:CD001877. [PMID: 23737396] doi:10.1002/14651858.CD001877.pub5

37. Skaane P, Bandos Al, Gullien R, Eben EB, Ekseth U, Haakenaasen $\mathrm{U}$, et al. Comparison of digital mammography alone and digital mammography plus tomosynthesis in a population-based screening program. Radiology. 2013;267:47-56. [PMID: 23297332] doi:10 .1148/radiol.12121373

38. Rose SL, Tidwell AL, Bujnoch LJ, Kushwaha AC, Nordmann AS, Sexton R Jr. Implementation of breast tomosynthesis in a routine screening practice: an observational study. AJR Am J Roentgenol. 2013;200:1401-8. [PMID: 23701081] doi:10.2214/AJR.12.9672

39. Hubbard RA, Kerlikowske K, Flowers Cl, Yankaskas BC, Zhu W, Miglioretti DL. Cumulative probability of false-positive recall or biopsy recommendation after 10 years of screening mammography: a cohort study. Ann Intern Med. 2011;155:481-92. [PMID: 22007042] doi:10.7326/0003-4819-155-8-201110180-00004

40. Carter JL, Coletti RJ, Harris RP. Quantifying and monitoring overdiagnosis in cancer screening: a systematic review of methods. BMJ. 2015;350:g7773. [PMID: 25569206] doi:10.1136/bmj.g7773

41. Pisano ED, Gatsonis C, Hendrick E, Yaffe M, Baum JK, Acharyya $S$, et al; Digital Mammographic Imaging Screening Trial (DMIST) Investigators Group. Diagnostic performance of digital versus film mammography for breast-cancer screening. N Engl J Med. 2005; 353:1773-83. [PMID: 16169887]

42. Skaane P, Bandos Al, Eben EB, Jebsen IN, Krager M, Haakenaasen $U$, et al. Two-view digital breast tomosynthesis screening with synthetically reconstructed projection images: comparison with digital breast tomosynthesis with full-field digital mammographic images. Radiology. 2014;271:655-63. [PMID: 24484063] doi:10.1148 /radiol.13131391

43. Berg WA, Zhang Z, Lehrer D, Jong RA, Pisano ED, Barr RG, et al; ACRIN 6666 Investigators. Detection of breast cancer with addition of annual screening ultrasound or a single screening MRI to mammography in women with elevated breast cancer risk. JAMA. 2012; 307:1394-404. [PMID: 22474203] doi:10.1001/jama.2012.388

44. Coldman A, Phillips N, Wilson C, Decker K, Chiarelli AM, Brisson $\mathrm{J}$, et al. Pan-Canadian study of mammography screening and mortality from breast cancer. J Natl Cancer Inst. 2014;106. [PMID: 25274578] doi:10.1093/jnci/dju261
45. Hellquist BN, Duffy SW, Abdsaleh S, Björneld L, Bordás P, Tabár $L$, et al. Effectiveness of population-based service screening with mammography for women ages 40 to 49 years: evaluation of the Swedish Mammography Screening in Young Women (SCRY) cohort. Cancer. 2011;117:714-22. [PMID: 20882563] doi:10.1002/cncr.25650 46. Barratt A. Overdiagnosis in mammography screening: a 45 year journey from shadowy idea to acknowledged reality. BMJ. 2015;350: h867. [PMID: 25736426] doi:10.1136/bmj.h867

47. Friedewald SM, Rafferty EA, Rose SL, Durand MA, Plecha DM, Greenberg JS, et al. Breast cancer screening using tomosynthesis in combination with digital mammography. JAMA. 2014;311:2499507. [PMID: 25058084] doi:10.1001/jama.2014.6095

48. Lee CH, Dershaw DD, Kopans D, Evans P, Monsees B, Monticciolo $D$, et al. Breast cancer screening with imaging: recommendations from the Society of Breast Imaging and the ACR on the use of mammography, breast MRI, breast ultrasound, and other technologies for the detection of clinically occult breast cancer. J Am Coll Radiol. 2010;7:18-27. [PMID: 20129267] doi:10.1016/j.jacr.2009.09.022 49. American College of Obstetricians-Gynecologists. Practice bulletin no. 122: Breast cancer screening. Obstet Gynecol. 2011;118: 372-82. [PMID: 21775869] doi:10.1097/AOG.0b013e31822c98e5

50. Oeffinger KC, Fontham ET, Etzioni R, Herzig A, Michaelson JS, Shih YC, et al; American Cancer Society. Breast cancer screening for women at average risk: 2015 guideline update from the American Cancer Society. JAMA. 2015;314:1599-614. [PMID: 26501536] doi: 10.1001/jama.2015.12783

51. Qaseem A, Snow V, Sherif K, Aronson M, Weiss KB, Owens DK; Clinical Efficacy Assessment Subcommittee of the American College of Physicians. Screening mammography for women 40 to 49 years of age: a clinical practice guideline from the American College of Physicians. Ann Intern Med. 2007;146:511-5. [PMID: 17404353]

52. American Academy of Family Physicians. Clinical Preventive Service Recommendation: Breast Cancer. Leawood, KS: American Academy of Family Physicians; 2016. Accessed at www.aafp.org /patient-care/clinical-recommendations/all/breast-cancer.html on 25 November 2015.

53. American Congress of Obstetricians and Gynecologists. WellWoman Recommendations. Washington, DC: American Congress of Obstetricians and Gynecologists; 2015. Accessed at www.acog.org /About-ACOG/ACOG-Departments/Annual-Womens-Health-Care Well-Woman-Recommendations on 25 November 2015.

54. Conray JA, Brown H. Well-Woman Task Force: components of the well-woman visit-executive summary. Obstet Gynecol. 2015; 126(4):697-701.

55. Tonelli $M$, Connor Gorber S, Joffres $M$, Dickinson J, Singh $H_{\text {, }}$ Lewin G, et al; Canadian Task Force on Preventive Health Care. Recommendations on screening for breast cancer in average-risk women aged 40-74 years. CMAJ. 2011;183:1991-2001. [PMID: 22106103] doi:10.1503/cmaj.110334

56. Giordano L, von Karsa L, Tomatis M, Majek O, de Wolf C, Lancucki L, et al; Eunice Working Group. Mammographic screening programmes in Europe: organization, coverage and participation. J Med Screen. 2012;19 Suppl 1:72-82. [PMID: 22972813]

57. Lauby-Secretan B, Scoccianti C, Loomis D, Benbrahim-Tallaa L, Bouvard V, Bianchini F, et al. Breast-cancer screening-viewpoint of the IARC Working Group. New Engl J Med. 2015;372:2353-8.

58. Patient Protection and Affordable Care Act. 42 U.S.C. $§ 2713$ (2010). 59. Siu AL, Bibbins-Domingo K, Grossman DC. Evidence-based clinical prevention in the era of the Patient Protection and Affordable Care Act: the role of the US Preventive Services Task Force. JAMA. 2015;314:2021-2. [PMID: 26421503] doi:10.1001/jama.2015.13154 60. Consolidated Appropriations Act. 42 U.S.C. § 9(h)(v)(229) (2015). 61. Sabatino SA, White MC, Thompson TD, Klabunde CN. Cancer screening test use-United States, 2013. MMWR Morb Mortal Wkly Rep. 2015;64:464-8. [PMID: 25950253]

62. National Committee for Quality Assurance. State of Health Care Quality: Breast Cancer Screening. 2015. Accessed at http://www.ncqa.org/ReportCards/HealthPlans/StateofHealth CareQuality/2015TableofContents/BreastCancer.aspx on 21 December 2015. 


\section{Annals of Internal Medicine}

\section{APPENDIX: MEMBERS OF THE USPSTF}

Members of the USPSTF at the time this recommendation was finalized $†$ are Albert L. Siu, MD, MSPH, Chair (Mount Sinai School of Medicine, New York, and James J. Peters Veterans Affairs Medical Center, Bronx, New York); Kirsten Bibbins-Domingo, PhD, MD, MAS, Co-Vice Chair (University of California, San Francisco, San Francisco, California); David C. Grossman, MD, $\mathrm{MPH}, \mathrm{Co}-$ Vice Chair (Group Health Research Institute, Seattle, Washington); Linda Ciofu Baumann, PhD, RN, APRN (University of Wisconsin, Madison, Wisconsin); Karina W. Davidson, PhD, MASc (Columbia University, New York, New York); Mark Ebell, MD, MS (University of Georgia, Athens, Georgia); Francisco A.R. García, MD, MPH (Pima County Department of Health, Tucson, Arizona); Matthew Gillman, MD, SM (Harvard Medical School and Harvard Pilgrim Health Care Institute, Boston, Massachusetts); Jessica Herzstein, MD, MPH (Independent Consultant, Washington, DC); Alex R. Kemper,
MD, MPH, MS (Duke University, Durham, North Carolina); Alex H. Krist, MD, MPH (Fairfax Family Practice, Fairfax, and Virginia Commonwealth University, Richmond, Virginia); Ann E. Kurth, PhD, RN, MSN, MPH (New York University, New York, New York); Douglas K. Owens, MD, MS (Veterans Affairs Palo Alto Health Care System, Palo Alto, and Stanford University, Stanford, California); William R. Phillips, MD, MPH (University of Washington, Seattle, Washington); Maureen G. Phipps, MD, MPH (Brown University, Providence, Rhode Island); and Michael P. Pignone, MD, MPH (University of North Carolina, Chapel Hill, North Carolina). Michael LeFevre, MD, MSPH, Immediate Past Chair (University of Missouri, Columbia, Missouri), also contributed to the development of this recommendation.

$\dagger$ For a list of current USPSTF members, go to www.uspreventiveservicestaskforce.org/Page/Name lour-members.

\begin{tabular}{|c|c|c|}
\hline A & $\begin{array}{l}\text { The USPSTF recommends the service. There is high certainty that } \\
\text { the net benefit is substantial. }\end{array}$ & Offer/provide this service. \\
\hline B & $\begin{array}{l}\text { The USPSTF recommends the service. There is high certainty that } \\
\text { the net benefit is moderate or there is moderate certainty that } \\
\text { the net benefit is moderate to substantial. }\end{array}$ & Offer/provide this service. \\
\hline C & $\begin{array}{l}\text { The USPSTF recommends selectively offering or providing this } \\
\text { service to individual patients based on professional judgment } \\
\text { and patient preferences. There is at least moderate certainty that } \\
\text { the net benefit is small. }\end{array}$ & $\begin{array}{l}\text { Offer/provide this service for selected patients depending on individual } \\
\text { circumstances. }\end{array}$ \\
\hline D & $\begin{array}{l}\text { The USPSTF recommends against the service. There is moderate or } \\
\text { high certainty that the service has no net benefit or that the } \\
\text { harms outweigh the benefits. }\end{array}$ & Discourage the use of this service. \\
\hline I statement & $\begin{array}{l}\text { The USPSTF concludes that the current evidence is insufficient to } \\
\text { assess the balance of benefits and harms of the service. Evidence } \\
\text { is lacking, of poor quality, or conflicting, and the balance of } \\
\text { benefits and harms cannot be determined. }\end{array}$ & $\begin{array}{l}\text { Read the Clinical Considerations section of the USPSTF Recommendation } \\
\text { Statement. If the service is offered, patients should understand the } \\
\text { uncertainty about the balance of benefits and harms. }\end{array}$ \\
\hline
\end{tabular}

\begin{tabular}{|c|c|}
\hline \multicolumn{2}{|c|}{ Appendix Table 2. USPSTF Levels of Certainty Regarding Net Benefit } \\
\hline Level of Certainty* & Description \\
\hline High & $\begin{array}{l}\text { The available evidence usually includes consistent results from well-designed, well-conducted studies in } \\
\text { representative primary care populations. These studies assess the effects of the preventive service on health } \\
\text { outcomes. This conclusion is therefore unlikely to be strongly affected by the results of future studies. }\end{array}$ \\
\hline Moderate & $\begin{array}{l}\text { The available evidence is sufficient to determine the effects of the preventive service on health outcomes, but } \\
\text { confidence in the estimate is constrained by such factors as: } \\
\text { the number, size, or quality of individual studies; } \\
\text { inconsistency of findings across individual studies; } \\
\text { limited generalizability of findings to routine primary care practice; and } \\
\text { lack of coherence in the chain of evidence. } \\
\text { As more information becomes available, the magnitude or direction of the observed effect could change, and this } \\
\text { change may be large enough to alter the conclusion. }\end{array}$ \\
\hline Low & $\begin{array}{l}\text { The available evidence is insufficient to assess effects on health outcomes. Evidence is insufficient because of: } \\
\text { the limited number or size of studies; } \\
\text { important flaws in study design or methods; } \\
\text { inconsistency of findings across individual studies; } \\
\text { gaps in the chain of evidence; } \\
\text { findings that are not generalizable to routine primary care practice; and } \\
\text { a lack of information on important health outcomes. } \\
\text { More information may allow an estimation of effects on health outcomes. }\end{array}$ \\
\hline
\end{tabular}

* The USPSTF defines certainty as "likelihood that the USPSTF assessment of the net benefit of a preventive service is correct." The net benefit is defined as benefit minus harm of the preventive service as implemented in a general primary care population. The USPSTF assigns a certainty level on the basis of the nature of the overall evidence available to assess the net benefit of a preventive service. 